\title{
Increased Emergency Department Computed Tomography Use for Common Chest Symptoms Without Clear Patient Benefits
}

\author{
Andrew S. Coco, MD, MS, and David T. O'Gurek, MD
}

Objective: The aim of this study was to examine changes in the utilization of computed tomography (CT) in the evaluation of common chest symptoms and the rate of clinically significant diagnoses in emergency departments after 2004.

Methods: This study analyzed the National Hospital Ambulatory Medical Care Survey, comparing 1997 to 1999 and 2005 to 2007. Set in US emergency departments, individuals older than 14 years old were eligible. The main outcome was proportion of common chest symptom-related visits $(\mathrm{n}=17,098)$ associated with a CT order before 2000 and after 2004. Secondary outcomes were the proportion of these visits associated with a clinically significant diagnosis (pulmonary embolism, acute myocardial infarction, acute coronary syndrome, heart failure, pneumonia, and pleural effusion); an incidental diagnosis such as lung mass; and a clinically nonsignificant diagnosis such as nonspecific chest pain.

Results: The proportion of common chest symptom-related visits associated with a CT order increased from $2.1 \%$ in 1997 to 1999 to $11.5 \%$ in 2005 to $2007(P<.001)$, whereas the overall proportion of these visits associated with a clinically significant diagnosis decreased from $23.6 \%$ in 1997 to 1999 to $19.1 \%$ in 2005 to $2007(P<.001)$. The rate of acute myocardial infarction diagnosis decreased from $6.6 \%$ to $3.3 \%(P<.001)$, whereas the rate of pulmonary embolism diagnosis did not change $(0.33 \%$ vs. $0.47 \% ; P=.24)$ from 1997 to 1999 to 2005 to 2007 . The rate of incidental diagnoses did not change $(0.13 \%$ vs. $0.17 \% ; P=.69)$, whereas the rate of clinically nonsignificant diagnoses increased from $35.6 \%$ to $45.8 \%(P<.001)$ from 1997 to 1999 to 2005 to 2007.

Conclusions: CT ordering in emergency departments for the evaluation of common chest symptoms has increased dramatically without improving the rate of pulmonary embolism or other clinically significant diagnoses. Overuse of CT exposes patients to radiation and increases health care costs without any apparent diagnostic benefit. (J Am Board Fam Med 2012;25:33-41.)

Keywords: Computed Tomography, Emergency Treatment, Radiation

Computed tomography (CT), although a useful diagnostic tool, is not without potential risks when overly utilized. Several studies suggest that, overall,

This article was externally peer reviewed.

Submitted 3 February 2011; revised 14 September 2011; accepted 23 September 2011.

From Lancaster General Research Institute and the Department of Family and Community Medicine, Lancaster General Health, Lancaster, PA.

Funding: none.

Conflict of interest: none declared.

Disclaimer: The authors had full access to all the data in the study and take responsibility for the integrity of the data and the accuracy of the data analysis.

Corresponding author: David T. O'Gurek, MD, Department of Family \& Community Medicine, Lancaster General Health, 540 N. Duke Street, 3rd Floor, Lancaster, PA 17604 (E-mail: dogurek2@lghealth.org). the number of CT scans ordered since the early 1990s has increased greater than 3-fold, likely, in part, because of its widespread availability as well as the rapidity with which an evaluation can be completed. ${ }^{1,2}$

With 10 million of the more than 110 million patients seeking care in emergency Departments in the United States annually who present with complaints of dyspnea, chest pain, or both, ${ }^{3}$ physicians are challenged with the daunting task of evaluating patients with symptoms most concerning for acute coronary syndrome, acute aortic syndrome, or pulmonary embolism (PE). Most patients presenting with these symptoms do not have a life-threatening condition; however, they often require additional testing to evaluate for these concerning conditions. 
Concern for acute aortic syndrome or PE often leads to CT ordering. Interestingly enough, of patients who present to emergency departments with complaints of chest pain, PE and aortic dissection were diagnosed only $0.4 \%$ and $0.3 \%$ of the time, respectively. $^{4}$

One study looking at trends at an academic medical center suggested that the increased use of chest CT scans has enabled earlier recognition of $\mathrm{PE}^{5}$; however, controversy exists over whether all identified patients had clinically significant lesions requiring anticoagulation. A more recent study showed that $24 \%$ of patients who had a CT ordered to evaluate for PE had a clinically significant incidental finding diagnosis and $33 \%$ had findings that supported an alternative diagnosis. ${ }^{6}$ However, an accompanying editorial concluded that the data also could be interpreted as demonstrating that CTs have been adopted too quickly, without adequate consideration of the benefits and risks. ${ }^{7}$

The risks of radiation and contrast exposure are important considerations in CT scan utilization. ${ }^{8,9}$ Patients are particularly at risk for contrast-induced nephropathy and cancer of the breast, thyroid, and lung. ${ }^{10}$ Given these factors, as well as additional costs to patients and the health care system, ${ }^{11}$ it is important to document the extent of increased CT utilization for common chest symptoms and its effect on diagnostic patterns.

We analyzed the National Hospital Ambulatory Medical Care Survey (NHAMCS) to determine changes in utilization of CT for common chest symptoms as well as changes in the rates of associated diagnoses after 2004.

\section{Methods}

\section{Study Design and Administration}

Data for this study was compiled from the 1997 to 1999 and 2005 to 2007 NHAMCS. The survey is administered by the National Center for Health Statistics (NCHS) for the Centers for Disease Control and Prevention. It is designed to meet the need for objective, reliable information about ambulatory medical care services in hospital outpatient and emergency departments in the United States.

The NHAMCS measures utilization and provision of ambulatory care services at US hospitals. Using a 4-stage probability sample design, NHAMCS collects a nationally representative sample of all visits to hospital outpatient depart- ments and emergency departments based in general and short-stay hospitals, excluding federal hospitals. Only data from emergency departments was used in this study. NHAMCS data are collected by hospital staff members and monitored by field representatives. Visit information is collected during a randomly assigned 4-week reporting period. The basic sampling unit used throughout the survey is patient visit. The sample of hospitals is randomly divided into subsets of equal size, and each subset is granted a 4-week period in a cycle such that each subset is not measured at the same time each year and that each subset is not measured in a given year. The NCHS institutional review board approved the protocol for the NHAMCS, including a waiver of the requirement for informed consent. More detailed description of the NHAMCS methodology is available from the NCHS. ${ }^{12}$

\section{Study Sample: Episodes of Care Associated with a Common Chest Symptom}

Up to 3 "complaints, symptoms, or other reason(s) for visit" are abstracted as free text and then coded centrally using a standard reason for visit classification (RVC) system. ${ }^{12}$ Visits with symptoms of chest pain (RCV code 1050), shortness of breath (RCV code 1415), and labored breathing (RCV code 1420) were identified and coded as a CC symptom-related visit.

\section{Covariates}

Patient age of 15 years and older (collapsed to 15-44 years, 45-64 years, $\geq 65$ years); sex; race (categories collapsed to white, black, and other); and insurance status (categories collapsed to private, Medicare, Medicaid, or self pay/other) are recorded for each visit. Geographic region is recorded as well.

\section{Comparative Time Periods}

Visits associated with a CT order were available from 1997 to 1999 and 2005 to 2007. From 2000 to 2004, CT ordering was combined with magnetic resonance imaging (MRI) ordering and not available as an individual variable. The data were stratified into two 3-year periods: 1997 to 1999 and 2005 to 2007. To assess for increasing CT utilization within the 2005 to 2007 study period, six 6-month time periods were created for a trend analysis: January-June and July-December for each of the 2 years. 
Primary Outcome: Proportion of Common Chest Symptom-Related Visits Associated With a CT Order

Visits were included that were coded with a positive response to the survey item computed axial tomography scan (1997 to 1999) or CT scan (2005 to 2006) or any CT scan (2007) under the question, Were any diagnostic/screening services ordered or provided during this visit?

\section{Secondary Outcomes: Common Chest Diagnoses}

Up to 3 diagnoses are recorded for each visit as free text and then coded by survey staff using the International Classification of Diseases, Clinical Modification, Ninth Revision (ICD-9-CM). ${ }^{13}$ Clinically significant CC diagnoses included acute myocardial infarct (ICD-9-CM code 410.0), acute coronary syndrome (ICD-9-CM code 411.0), pulmonary embolism and infarction (ICD-9-CM code 415.1), heart failure (ICD-9-CM code 428.0), pneumonia, organism unspecified (ICD-9-CM code 486), and unspecified pleural effusion (ICD-9-CM code 511.9). The sample of visits associated with diagnoses of aortic dissection, cardiomegaly, pericarditis, emphysema, or hiatal hernia was not adequate for evaluation.

Incidental findings included diagnoses of intrathoracic lymph nodes (ICD-9-CM code 196.1), swelling, mass, or lump in chest (ICD-9-CM code 786.6), abdominal mass (ICD-9-CM code 789.3), and coin lesion lung (ICD-9-CM code 793.1). Clinically nonspecific diagnoses included general symptoms (ICD9-CM code 780) and respiratory system/other chest symptoms (ICD-9-CM code 786).

\section{Proportion of Common Chest Symptom-Related Visits Associated With Another Diagnostic Imaging Order}

Under the survey question, Were any diagnostic/ screening services ordered or provided at this visit?:

1. Radiograph: Visits were included that were coded with a positive response to the survey item "chest radiograph, extremity radiograph, or other radiograph" (1997-1999); or "radiograph" (2005-2007).

2. Ultrasound: Visits were included that were coded with a positive response to the survey item "ultrasound" (1997-1999) and (2005-2007).

3. MRI: Visits were included that were coded with a positive response to the survey item "MRI" (1997-1999 and 2005-2006) or "any MRI" (2007).
4. Other diagnostic image (other than CT, radiograph, ultrasound, or MRI): Visits were included that were coded with a positive response to the survey item "other diagnostic imaging" (1997-1999) or "other imaging" (2005-2007). Ventilation-perfusion scans are included, but not differentiated, in this category.

\section{Data Analysis}

The weights, strata, and primary sampling unit design variables provided by NCHS were used for all analyses. The proportion of CC symptom-related visits associated with a CT order, a clinically significant diagnosis, a clinically nonsignificant diagnosis, and with another diagnostic imaging order were evaluated with the $\chi^{2}$ test. Linear regression was used to test for time trends, with calendar year included as a linear predictor. A multivariate logistic regression model was developed to predict associations with a CT order in CC symptom-related visits. All analyses used the svy command provided in Stata version 11 (StataCorp, College Station, Texas). All $P$ values are 2 -tailed; $P<.05$ was considered statistically significant.

\section{Results}

During the 6 survey years (1997-1999 and 20052007), NHAMCS collected data on 136,751 emergency department visits for individuals older than 14 years of age: 53,145 in 1997 to 1999 and 83,606 in 2005 to 2007 . A CC symptom-related RVC code was recorded for 17,098 of these visits. Using survey weights and averaging over our study period, we estimated that 11 million (95\% CI, 10-12 million) CC symptom-related visits were made annually to US emergency departments, representing $13.0 \%$ (95\% CI, $12.7 \%-13.4 \%$ ) of all emergency department visits within the age criteria. The proportion of CC symptom-related visits was similar in 1997 to 1999 and 2005 to 2007 (12.9\% vs $13.3 \%$; $P=.22)$.

Whites made $76 \%$ of all CC symptom-related visits; blacks, 21\%; and Asians/others, 3\% (Table 1). Patients making CC symptom-related visits in 1997 to 1999 were more likely to be older than 65 years old and have private or Medicare insurance compared with those patients making visits in 2005 to 2007 . Other differences in visit and patient characteristics were not large. 
Table 1. Characteristics of Common Chest Symptom-Related* Visits to United States Emergency Departments, 1997 to 1999 and 2005 to $2007(\mathrm{n}=17,098)$

\begin{tabular}{|c|c|c|c|c|}
\hline Characteristic & $\begin{array}{l}\text { Overall Proportion } \\
\text { of Visits (\%) }\end{array}$ & $\begin{array}{l}\text { Proportion of Visits, } \\
1997 \text { to } 1999(\mathrm{n}=6,851)(\%)\end{array}$ & $\begin{array}{l}\text { Proportion of Visits, } \\
2005 \text { to } 2007(\mathrm{n}=10,247)(\%)\end{array}$ & $P$ \\
\hline \multicolumn{5}{|l|}{ Sex } \\
\hline Female & 55 & 54 & 56 & \multirow{2}{*}{.17} \\
\hline Male & 45 & 46 & 44 & \\
\hline \multicolumn{5}{|l|}{ Age (years) } \\
\hline $15-44$ & 38 & 37 & 39 & \multirow{3}{*}{$<.01$} \\
\hline $45-64$ & 30 & 28 & 33 & \\
\hline$>65$ & 32 & 35 & 29 & \\
\hline \multicolumn{5}{|l|}{ Race } \\
\hline White & 76 & 78 & 74 & \multirow{3}{*}{.04} \\
\hline Black & 21 & 20 & 23 & \\
\hline Other & 3 & 2 & 3 & \\
\hline \multicolumn{5}{|l|}{ Insurance } \\
\hline Private & 35 & 36 & 33 & \multirow{4}{*}{$<.01$} \\
\hline Medicare & 31 & 34 & 29 & \\
\hline Medicaid & 17 & 13 & 20 & \\
\hline Self-pay/other & 17 & 17 & 17 & \\
\hline \multicolumn{5}{|l|}{ Geographic region } \\
\hline Northeast & 20 & 21 & 19 & \multirow{4}{*}{.65} \\
\hline Midwest & 24 & 26 & 22 & \\
\hline South & 38 & 35 & 41 & \\
\hline West & 18 & 18 & 18 & \\
\hline
\end{tabular}

${ }^{*}$ Common chest symptoms are chest pain, shortness of breath, or labored breathing.

These sample records represented a total of 4829,656 CC symptom-related visits associated with a CT order, of which 654,518 (95\% CI, $527,568-781,467)$ occurred in 1997 to 1999 and 4,175,138 (95\% CI, 3543,498-4,806,778) occurred in 2005 to 2007.

\section{CT Utilization}

The proportion of CC symptom-related visits associated with a CT order was significantly higher in 2005 to 2007 for each individual CC symptom as well as for all symptoms combined, which increased from $2.1 \%$ in 1997 to 1999 to $11.5 \%$ in 2005 to $2007(P<.001)$ (Figure 1). Furthermore, the rate of CT ordering almost doubled from $8.6 \%$ in the first 6 months of 2005 to $15.8 \%$ in the last 6 months of 2007 (Figure 2).

\section{Symptom-Related Diagnoses}

When adjusted for the demographic variables in Table 1, CC symptom-related visits from 1997 to 1999 were more likely to be associated with a clinically significant diagnosis $(23.6 \%$ vs $19.1 \% ; P<$ .001) when compared with visits in 2005 to 2007
(Figure 3). Acute myocardial infarction was the largest contributing diagnosis to the rate discrepancy between time periods. In addition, among the 17,098 visits associated with CC symptoms, a total of 82 encounter records, or $0.41 \%$ (95\% CI, $0.30 \%-0.52 \%)$, showed an association with a $\mathrm{PE}$ diagnosis. The proportion of CC symptom-related visits associated with a $\mathrm{PE}$ diagnosis did not change between study periods, varying from $0.33 \%$ in 1997 to 1999 to $0.47 \%$ in 2005 to $2007(P=.21)$. There were 30 of 6851 CC symptom-related visits $(0.38 \%)$ associated with an incidental diagnosis in 1997 to 1999 and 55 of 10,247 of these visits (0.49\%) in 2005 to 2007 ( $P=.18)$. CC symptomrelated visits from 2005 to 2007 were more likely to be associated with the clinically nonsignificant diagnosis $(45.8 \%$ vs $35.6 \% ; P<.001)$ when compared with visits in 1997 to 1999.

\section{Other Diagnostic Imaging}

CC symptom-related visits after 2004 were as likely to be associated with a radiograph order (68.4\% vs $66.0 \% ; P=.09)$ and more likely to be associated with an ultrasound order $(1.8 \%$ vs $1.0 \%$; 
Figure 1. Comparison of the proportion of common chest (CC) symptom-related visits associated with computed tomography orders in US emergency departments between 1997 to 1999 and 2005 to 2007 . $P<.0001$ for all comparisons. *Individual symptom totals do not total overall CC symptom-related visit total because some visits were associated with one than one CC symptom.

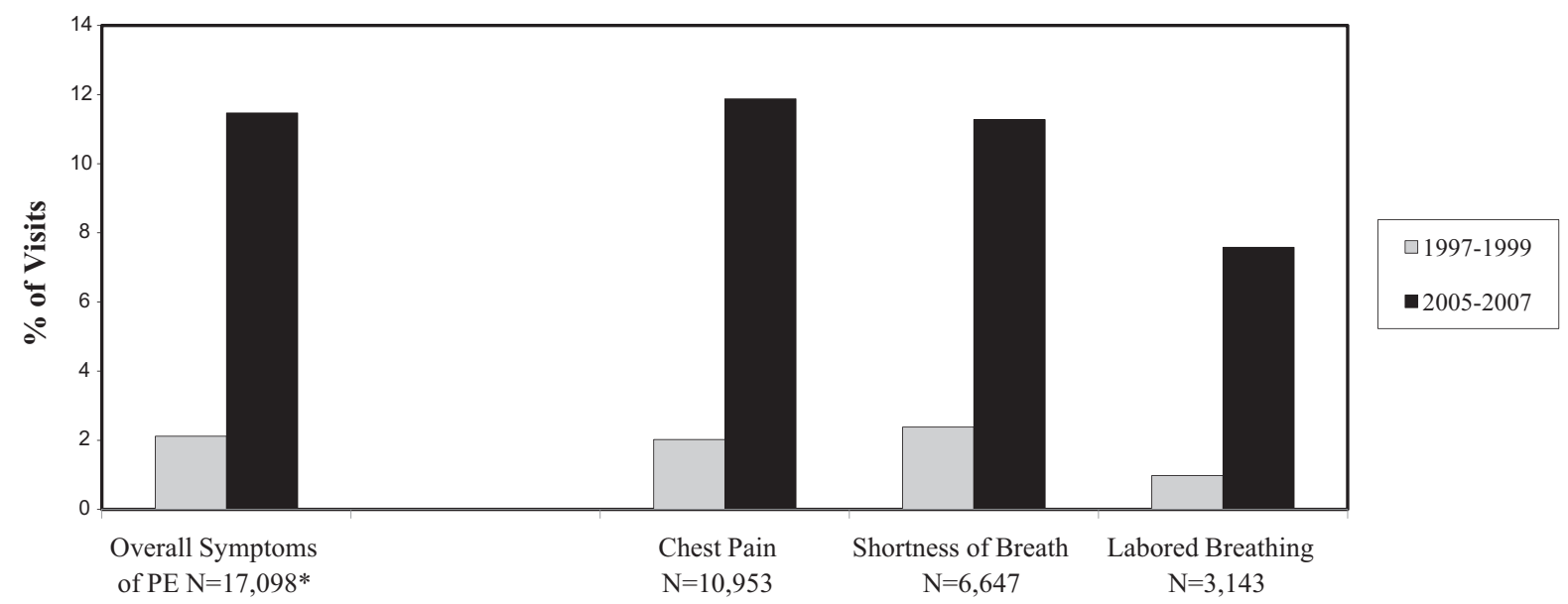

Symptom

$P<.01)$, other image order $(2.6 \%$ vs $1.9 \% ; P=$ $.04)$ or MRI order $(0.3 \%$ vs $0.1 \% ; P=.02)$ when compared with visits in 1997 to 1999.

\section{Associations With CT Order}

In multivariable logistic regression modeling, independent predictors of a CC symptom related-visit associated with a CT order were 2005 to 2007 time period versus 1997 to 1999 time period, age more than 65 years versus age 15 to 44 years, Medicaid versus private insurance, and western region versus northeast region (Table 2).

\section{Discussion}

In recent years, patients presenting to US emergency departments with CC symptoms were almost 6.5 times more likely to have undergone a CT examination, and by 2007 the likelihood had increased further. In many ways this is not surprising given the advancements in multidetector technology, rapidity of the test, and widespread access. A rise in the prevalence of certain conditions and illnesses enhanced by the utilization of imaging may be expected given this increased utilization.

Figure 2. Trend in the proportion of common chest (CC) symptom-related visits associated with a computed tomography order in US emergency departments, 2005 to $2007 . P<.001$ for trend.

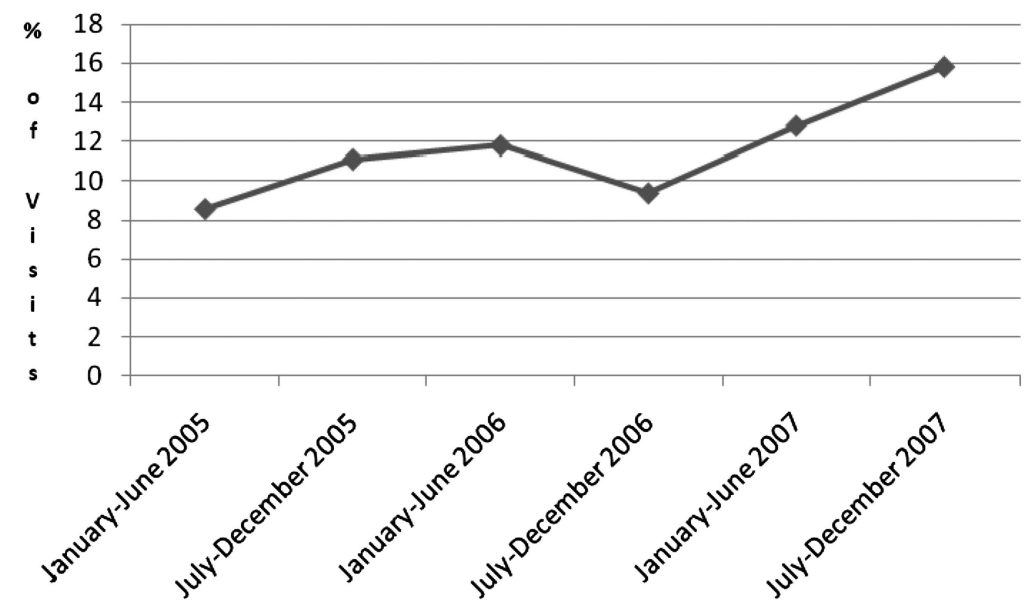


Figure 3. Comparison of proportion of diagnostic categories of CC symptom-related visits in US emergency departments between 1997 to 1999 and 2005 to 2007 ( $(\mathrm{n}=17,098)$. Results have been adjusted for sex, age, race, insurance, and region.

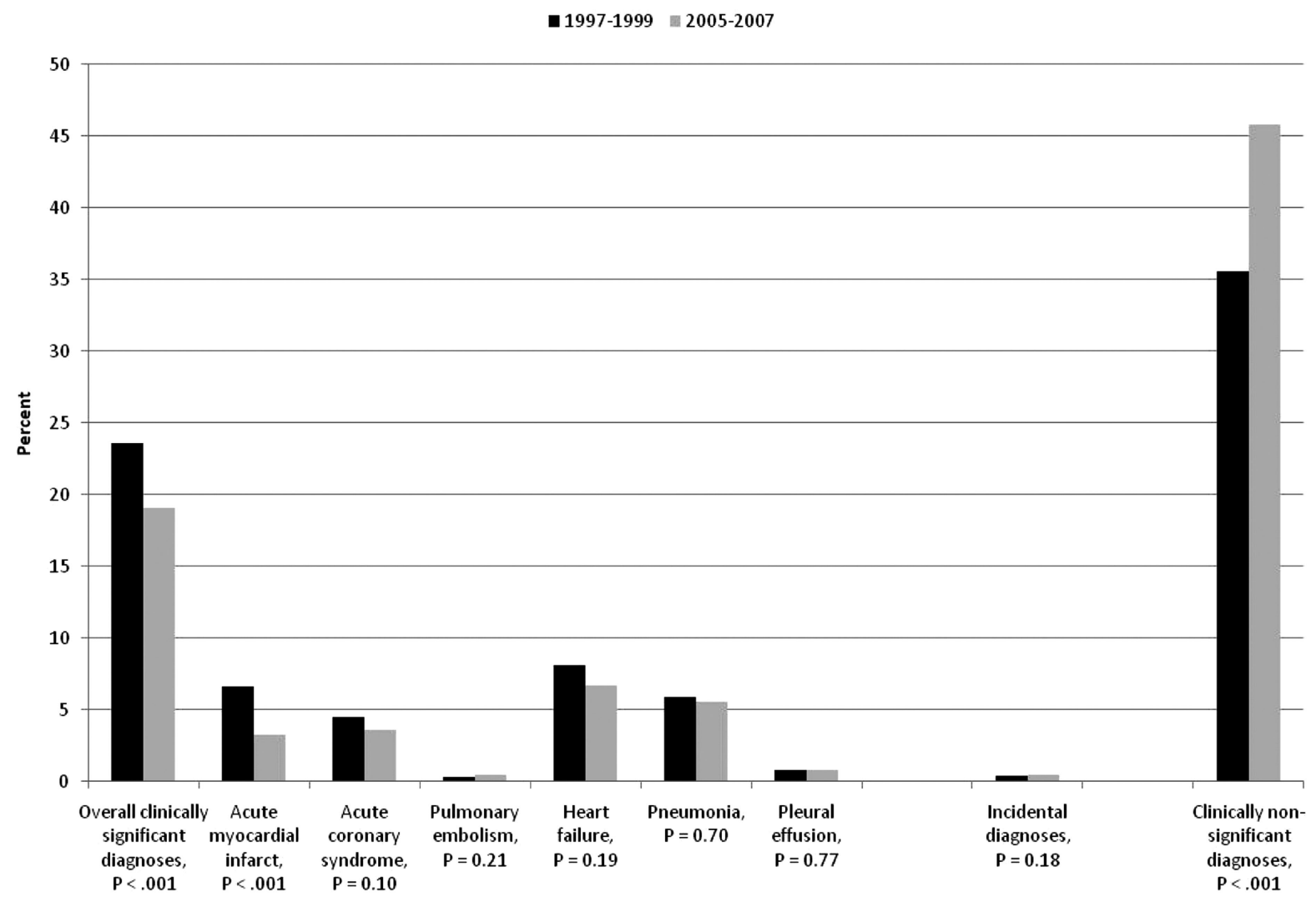

First, such an escalating rate of CT utilization might be expected to result in a commensurate increase in $\mathrm{PE}$ diagnoses, as suggested by prior literature. Somewhat unpredictably, our results do not support this assumption; the rate of $\mathrm{PE}$ diagnosis did not change from the time period before 2000 to the time period after 2004, results that are similar to a single institution study where a drop in PE detection occurred. ${ }^{14}$ This, however, markedly differs from a previous study that suggests that CT, through earlier diagnosis of $\mathrm{PE}$, has reduced associated morbidity and mortality. ${ }^{1}$ Instead, our results point to CTs being ordered unselectively for patients with chest pain or breathing problems in 2005 to 2007 , as evidenced by the increase in the nonspecific diagnoses of respiratory system/other chest symptoms and general symptoms.

In addition, the high resolution associated with increased CT utilization also has been demonstrated in previous studies to diagnose an increased number of pneumonias over chest radiograph alone. ${ }^{15,16}$ Our study does not reproduce these findings, with pneumonia being diagnosed as often despite increased utilization in CT. With increases in the number of nonspecific diagnoses and no improvements in clinically significant diagnoses such as $\mathrm{PE}$ and pneumonia among patients presenting with CC symptoms, increased utilization seems to provide little to add in the diagnostic work-up and certainly is not altering treatment in the setting of inappropriate ordering.

Furthermore, with the escalating rates of CT utilization, a high number of clinically significant incidental findings have been reported, ${ }^{6}$ which has led some physicians to argue that $\mathrm{CT}$ is useful in patients even when clinical suspicion of more concerning diagnoses (eg, PE) is high. ${ }^{17,18}$ However, our results differ from previous studies in that no significant change in incidental findings was discovered during the period of increased CT utilization. Our results are not directly comparable with other findings, however, because they probably represent a lower risk population and are derived from a national sample versus a single institution where 
Table 2. Multivariate Logistic Regression Model Predicting Common Chest Symptom-Related* Visits Associated with a Computed Tomography Order in United States Emergency Departments, 1997 to 1999 and 2005 to 2007

\begin{tabular}{ll}
\hline Characteristic & $\begin{array}{c}\text { Odds Ratio }{ }^{\dagger}(95 \% \text { CI }) \text { of a Computed } \\
\text { Tomography Order }\end{array}$ \\
\hline Time period & \\
$1997-1999$ & $1.00(-)$ \\
$2005-2007$ & $6.41(5.12-8.03)$ \\
Sex & $1.00(-)$ \\
Female & $0.92(0.80-1.07)$ \\
Male & \\
Age (years) & $1.00(-)$ \\
$15-44$ & $1.09(0.92-1.29)$ \\
$45-64$ & $1.30(1.04-1.61)$ \\
$>65$ & $1.00(-)$ \\
Race & $0.92(0.77-1.11)$ \\
White & $0.73(0.50-1.07)$ \\
Black & \\
Other & $1.00(-)$ \\
Health insurance & $0.88(0.70-1.10)$ \\
Private & $0.71(0.57-0.88)$ \\
Medicare & $0.96(0.77-1.19)$ \\
Medicaid & $1.00(-)$ \\
Self-pay/other & $1.24(0.90-1.70)$ \\
Geographic region & $0.99(0.74-1.31)$ \\
Northeast & $1.51(1.15-1.99)$ \\
Midwest & \\
South & \\
West & \\
\hline &
\end{tabular}

${ }^{*}$ Common chest symptoms are chest pain, shortness of breath, or labored breathing.

${ }^{\dagger}$ Adjusted for all other variables in the table.

radiologists may report differently because of the local medicolegal environment. Furthermore, incidental findings discovered on CT are not without their risks; the dose of radiation from a single CT, let alone repeated studies to follow pulmonary nodules, increases risk of radiation-induced cancer. ${ }^{19,20}$ There are also risks associated with repeated intravenous contrast, ${ }^{21}$ and an emotional burden can be placed on a patient being informed about a potential diagnosis of cancer. ${ }^{22}$ Interestingly enough, incidental pulmonary nodules found in patients receiving cardiac CT were found in 81 of 459 patients and, after 2 year follow-up, none developed lung cancer. $^{23}$

A 2004 Intersociety of Radiology summary report on inappropriate ordering suggests that increased utilization is likely secondary to poor un- derstanding of what study is needed, high public expectations, medical liability issues, and self referral. ${ }^{24}$ Subsequent studies largely have confirmed this opinion because patient self referral, ${ }^{25}$ the style and content of clinical education, ${ }^{26}$ and the fear of malpractice litigation ${ }^{27}$ have been shown to contribute to increased ordering. Somewhat troubling with regard to public expectations and self referral of patients is that an increasing number of diagnostic imaging studies, including CT scan, have transitioned from inpatient settings to outpatient centers and specialty hospitals. ${ }^{28,29}$ One study linked these centers with easier access for individuals with private insurances, a group that traditionally has been more willing to pay for advanced diagnostic procedures. ${ }^{30}$

In a health care system that is already struggling and overextended, the cost of unnecessary and repeat imaging creates undue stress. ${ }^{31}$ Medicare spending for imaging studies between 2000 and 2005 more than doubled, from $\$ 6.6$ billion to $\$ 13.7$ billion, which represents an growth rate twice that of physician fee schedules. ${ }^{32} \mathrm{With}$ particular regard to chest symptoms, evaluation of data from a single health plan demonstrated that chest CT was among a short list of anatomic sites with dramatic increases in cost from 1997 to $2006 .{ }^{33}$ The increased use of diagnostic imaging has led to a significant increase in associated costs, estimated to exceed $\$ 100$ billion per year in the United States. ${ }^{34}$

Until recent years, with expanding data on the use of CT scans, heightened awareness of the risks of radiation exposure with CT was not well appreciated by physicians ordering and reading these studies. Physicians were largely unaware of the dose of radiation and concomitant associated cancer risks. ${ }^{35,36}$ A longitudinal study of workers in the nuclear industry, exposed to a radiation dose similar to a standard CT, demonstrated increased mortality from cancer. ${ }^{37}$ These data are particularly troublesome, given that a large number of individuals receiving CT scans are middle aged, among whom the risks of exposure and re-exposure to radiation could become manifest over time. Further studies are demonstrating increased cancer risk, ${ }^{38,39}$ with particular concern in younger adults and the pediatric population. A recent study using risk models has suggested that approximately 29,000 future cancers could be related to CT scans performed in the US in 2007 alone. ${ }^{40}$ The question of whether the benefits previously thought to out- 
weigh the burdens associated with CT remains unanswered; however, with known risks, some suggest that the elements of informed consent be met by communicating these possible risks of the diagnostic procedure. ${ }^{41}$

There were some aspects of the data that may limit the conclusions that can be drawn from our results. First, until the last year of the study (2007), the data did not specify anatomic sites associated with CT orders. So, although we assumed visits associated with chest symptoms were associated with chest CT orders, it is possible that some of the orders were for head or abdominal CT scans instead. This could have led to an overestimation of the increase in CT utilization. Another limitation related to this is the inability to distinguish CT angiography from other types of CT, again possibly leading to overestimation of the increase in CT utilization.

Newer technology and a heightened awareness of the risks of ordering unnecessary testing may lead to further adoption of evidence-based clinical decision-making pathways. Recent evidence suggests that hand-held clinical decision support systems may improve the diagnostic workup for PE, ${ }^{42}$ and the advent of electronic medical record technologies may allow for easier use of these tools.

\section{Conclusions}

This study provides evidence that CT utilization in the evaluation of CC symptoms has increased significantly over time without significant changes in the diagnosis of related disorders. Further studies are needed to investigate whether evidence-based clinical guidelines are being used and whether this affects the proportion of visits during which CT is ordered. With the radiation risks associated with CT becoming more apparent, utilization of health care resources from an evidential and public health-based approach is warranted.

\section{References}

1. Brenner, DJ, Hall EJ. Computed tomography-an increasing source of radiation exposure. N Engl J Med 2007;357:2277-84.

2. Mettler, FA, Thomadsen, BR, Bhargavan M, et al. Medical radiation exposure in the U.S. in 2006: preliminary results. Health Phys 2008;95(5):502-7.

3. McCaig LF. National Ambulatory Medical Care Survey: emergency department summary. Hyattsville, MD: National Center for Health Statistics; 2000;326:1-31.
4. Kohn MA, Kwan E, Gupta M, Tabas JA. Prevalence of acute myocardial infarction and other serious diagnoses in patients presenting to an urban emergency department with chest pain. J Emerg Med 2005;29(4):383-90.

5. Wittram C, Meehan MJ, Halpern EF, Shepard JA, McLoud TC, Thrall JH. Trends in thoracic radiology over a decade at a large academic medical center. J Thorac Imaging 2004;19(3):164-70.

6. Hall WB, Truitt SG, Scheunemann LP, et al. The prevalence of clinically relevant incidental findings on chest computed tomographic angiograms ordered to diagnose pulmonary embolism. Arch Intern Med 2009;169:1961-5.

7. Schnatter A. Computed tomographic pulmonary angiography to diagnose acute pulmonary embolism: the good, the bad and the ugly. Arch Intern Med 2009;169:1966-8.

8. Brenner DJ, Doll R, Goodhead DT, et al. Cancer risks attributable to low doses of ionizing radiation: assessing what we really know. Proc Natl Acad Sci U S A 2003;100:13761-6.

9. Fazel R, Krumholz HM, Wang Y, et al. Exposure to low-dose ionizing radiation from medical imaging procedures. N Engl J Med 2009;361(9):849-57.

10. Einstein AJ, Henzlova MJ, Rajagopalan S. Estimating risk of cancer associated with radiation exposure from 64-slice computed tomography coronary angiography. JAMA 2007;298(3):317-23.

11. Rothenberg BM, Korn A. The opportunities and challenges posed by the rapid growth of diagnostic imaging. J Am Coll Radiol 2005;2:407-10.

12. Centers for Disease Control and Prevention. Ambulatory health care data. Questionnaires, datasets, and related documentation. Survey methodology. Available at http://www.cdc.gov/nchs/ahcd/ahcd_ questionnaires.htm. Accessed October 23, 2009.

13. Centers for Disease Control and Prevention. Classification of diseases, functioning, and disability. International Classification of Disease, Ninth Revision, Clinical Modification. Available at http://www. cdc.gov/nchs/icd/icd $9 \mathrm{~cm} . h \mathrm{tm}$. Accessed November 20, 2009.

14. Donohoo JH. Utilization patterns and diagnostic yield of 3421 consecutive multidetector row computed tomography pulmonary angiograms in a busy emergency department. J Comput Assist Tomogr 2008;32:421-5.

15. Hayden GE, Wrenn KW. Chest radiograph vs. computed tomography scan in the evaluation for pneumonia. J Emerg Med 2009;36(3):266-70.

16. Syrjala H, Broas M, Suramo I, Ojala A, Lahde S. High-resolution computed tomography for the diagnosis of community-acquired pneumonia. Clin Infect Dis 1998;27(2):358-63.

17. Tsai KL, Gupta E, Haramati LB. Pulmonary atelectasis: a frequent alternative diagnosis in patients 
undergoing CT-PA for suspected pulmonary embolism. Emerg Radiol 2004;10(5):282-6.

18. van Strijen MJ, de Monyé W, Schiereck J, et al, Advances in New Technologies Evaluating the Localisation of Pulmonary Embolism Study Group. Single-detector helical computed tomography as the primary diagnostic test in suspected pulmonary embolism: a multicenter clinical management study of 510 patients. Ann Intern Med 2003;138(4):307-14.

19. Rixe J, Conradi G, Rolf A, et al. Radiation dose exposure of computed tomography coronary angiography: comparison of dual-source, 16-slice and 64slice CT. Heart 2009;95(16):1337-42.

20. Ghotbi N, Iwanaga M, Ohtsuru A, Ogawa Y, Yamashita S. Cancer screening with whole-body PET/CT for healthy asymptomatic people in Japan: re-evaluation of its test validity and radiation exposure. Asian Pac J Cancer Prev 2007;8(1):93-7.

21. Mitchell AM, Kline JA. Contrast nephropathy following computed tomography angiography of the chest for pulmonary embolism in the emergency department. J Thromb Haemost 2007;5(1):50-4.

22. Byrne MM, Weissfeld J, Roberts MS. Anxiety, fear of cancer, and perceived risk of cancer following lung cancer screening. Med Decis Making 2008;28(6):917-25.

23. Iribarren $C$, Hlatky MA, Chandra $M$, et al. Incidental pulmonary nodules on cardiac computed tomography: prognosis and use. Am J Med 2008;121(11):989-96.

24. Dunnick NR, Applegate K, Arenson RL. The inappropriate use of imaging studies: a report of the 2004 Intersociety Conference. J Am Coll Radiol 2005;2:401-6.

25. Medicare Payment Advisory Commission. Impact of physician self-referral on use of imaging services within an episode. In: Report to the Congressimproving incentives in the Medicare program. Washington, DC: Medicare Payment Advisory Commission; June 2009:81-96. Available at http:// www.medpac.gov/documents/Jun09_EntireReport. pdf. Accessed July 22, 2010.

26. Hillman BJ, Goldsmith JC. The uncritical use of hightech medical imaging. N Engl J Med 2010;363:4-6.

27. Massachusetts Medical Society. Investigation of defensive medicine in Massachusetts. Informational report I-08. Waltham, MA: Massachusetts Medical Society; November 2008. Available at http://www. ncrponline.org/PDFs/Mass_Med_Soc.pdf. Accessed July 22, 2010.

28. Iglehart JK. The emergence of physician-owned specialty hospitals. N Engl J Med 2005;352:78-84.

29. Guterman S. Specialty hospitals: a problem or a symptom? Health Aff (Millwood) 2006;25:95-105.

30. Mitchell JM. Utilization trends for advanced imaging procedures: evidence from individuals with pri- vate insurance coverage in California. Med Care 2008;46:460-6.

31. Piazza G, Goldhaber SZ. Chest computed tomography in the emergency department for suspected pulmonary embolism: it's time to practice what we preach. N Engl J Med 2009;54:49-52.

32. Medicare Payment Advisory Commission. A data book: healthcare spending and the Medicare program. June 2007. Available at http://www.aamc.org/ download/73232/data/. Accessed May 16, 2011.

33. Smith-Bindman R, Miglioretti DL, Larson EB. Rising use of diagnostic medical imaging in a large integrated health system. Health Aff 2008;27(6): 1491-502.

34. Iglehart JK. The new era of medical imaging: progress and pitfalls. N Engl J Med 2006;354(26): 2822-8.

35. Shiralkar S, Rennie A, Snow M, Galland RB, Lewis $\mathrm{MH}$, Gower-Thomas K. Doctor's knowledge of radiation exposure: questionnaire study. BMJ 2003; 327:371-2.

36. Lee CI, Haims AH, Monico EP, Brink JA, Forman HP. Diagnostic CT scans: assessment of patient, physician, and radiologist awareness of radiation dose and possible risks. Radiology 2004;231: 393-8.

37. Cardis E, Vrijheid M, Blettner M, et al. The 15country collaborative study of cancer risk among radiation workers in the nuclear industry: estimates of radiation-related cancer risks. Radiat Res 2007; 167:396-416.

38. Sodickson A, Baeyens PF, Andriole KP, et al. Recurrent CT, cumulative radiation exposure, and associated radiation-induced cancer risks from CT of adults. Radiology 2009;251(1):175-84.

39. Berrington de Gonzalez A, Mahesh M, Kim KP, et al. Projected cancer risks from computed tomographic scans performed in the United States in 2007. Arch Intern Med 2009;169(22):2071-7.

40. Rice HE, Frush DP, Farmer D, Waldhausen JH. Review of radiation risks from computed tomography: essentials for the pediatric surgeon. J Pediatr Surg 2007;42(4):603-7.

41. Karsli T, Kalra MK, Self JL, Rosenfield JA, Butler S, Simoneaux S. What physicians think about the need for informed consent for communicating the risk of cancer from low-dose radiation. Pediatr Radiol 2009; 39(9):917-25.

42. Roy PM, Durieux P, Gillaizeau F, et al. A computerized handheld decision-support system to improve pulmonary embolism diagnosis. Ann Intern Med 2009;151:677-86. 